\title{
Propiedades químicas y creencias curativas populares del Maguey o Cabuya (Agave americana L.) Caso, Churcampa, Huancavelica
}

\author{
Chemical properties and popular healing beliefs of agave (Agave americana L.) in \\ Churcampa - Huancavelica case
}

Elvis Pineda ${ }^{1}$, Dora Uribarri ${ }^{2}$

http://dx.doi.org/10.21503/CienciayDesarrollo.2014.v17i1.09

\section{RESUMEN}

Huancavelica es considerado dentro del mapa de pobreza uno de los departamentos más pobres del país, con problemas de primer orden desde la inseguridad alimentaria, hecho que se refleja en los altos índices de desnutrición crónica, daño acumulativo en el tiempo, que afecta a la población infantil; no obstante esta región ofrece una diversidad de recursos, que por la falta de apoyo técnico, económico, no elevan el nivel de productividad y/o comercialización.

Uno de los tantos recursos que posee es el Maguey o Cabuya (Agave americana L.), que tiene diversas formas de aprovechamiento que estamos estudiando, y que explotándolo de manera sostenida y bajo un criterio técnico ambiental podría mejorar la calidad de vida del poblador local.

Palabras Clave: Criterio técnico ambiental, cabuya.

\section{ABSTRACT}

Huancavelica is considered within the map of poverty, one of the poorest departments in the country, with problems of first order from food insecurity, which is reflected in high rates of chronic malnutrition, cumulative damage over time, affecting the child population; however, this region offers a variety of resources, which due to the lack of technical and economical support, not to raise the level of productivity or marketing.

One of the many resources that agave has (Agave americana L.) is the various forms of exploitation that we are studying, and exploited it in a sustained manner and under an environmental technical opinion could improve the quality of life of the local settler.

Keywords: Environmental technical opinion, agave.

\section{INTRODUCCIÓN}

Huancavelica es considerado dentro del mapa de pobreza uno de los departamentos más pobres del país, con problemas de primer orden desde la inseguridad alimentaria, hecho que se refleja en los altos índices de desnutrición crónica, daño acumulativo en el tiempo, que afecta a la población infantil; no obstante esta región ofrece una diversidad de recursos, que por la falta

\footnotetext{
1 Ingeniero, Consultor en Proyectos Productivos y Ambientales

2 Químico Farmacéutico, Magister, Docente UAP-Filial Ayacucho. E-mail: d_uribarri@uap.edu.pe
} 
de apoyo técnico, económico, no elevan el nivel de productividad y/o comercialización.

Uno de los tantos recursos que posee la provincia de Churcampa, departamento de Huancavelica, es el maguey o cabuya (Agave americana L.), que tiene diversas formas de aprovechamiento que estamos estudiando, y que explotándolo de manera sostenida y bajo un criterio técnico ambiental podría mejorar la calidad de vida del poblador local.

La Cabuya o Maguey (Agave americana L.) es una especie resistente a las sequías y se adaptan con facilidad a otros climas y tipos de suelos, encontrándose asociada a partir de los 2,500m de altitud a especies como la: Tara (Caesalpinea spinosa), Acacias, Molle (Schinus molle) y otros.

Es una planta de hojas en roseta, gruesas y carnosas, dispuestas sobre un tallo corto cuya piña inferior no sobresale de la tierra. Sus hojas están rígidas, con marginal y espinas del apical; guarda la insulina en el árbol y produce fructosa.
El uso de los agaves se remota en el Perú a la época precolombina, cuando los pueblo indígenas encontraron en esta planta una fuente abastecedora de materia prima para elaborar múltiples productos: de las pencas, obtenían hilos para tejer costales, morrales o pizcas, redes de pesca y cordeles; también enteras se usaban para techar las casas a modo de tejado; los troncos secos (tallo floral que alcanza más de tres metros), servían como vigas, como cercas para delimitar terrenos; las púas o espinas se utilizaban como clavos y como agujas; del jugo del maguey o upi, hervido y secado se obtiene la chancaca, pero en su estado fresco se bebe directamente de la planta y del cual se dice tiene propiedades curativas; pero también de el se puede elaborar otras bebidas, además del alcohol, como el tequila, el mezcal y vinagre. También es utilizado por los campesinos por sus propiedades para el engorde de los porcinos, que mezclan con la alimentación diaria (Como se observa en Ayacucho y Huancavelica). En la siguiente tabla resumimos las diferentes propiedades benéficas que nos otorga el maguey.

\section{Tabla 1. Productos potenciales a extraer industrialmente}

\begin{tabular}{|c|c|c|c|}
\hline Licor & Dulce & Fibra & Usos Medicinales \\
\hline $\begin{array}{c}\text { Upi } \\
\text { Tequila } \\
\text { Mescal } \\
\text { Vinagre }\end{array}$ & $\begin{array}{c}\text { Chancaca } \\
\text { Toffes o } \\
\text { Caramelos }\end{array}$ & $\begin{array}{l}\text { Para confeccionar } \\
\text { sogas, soguillas, } \\
\text { etc., en base a } \\
\text { las pencas secas } \\
\text { para hacer papel, } \\
\text { compost y humus. }\end{array}$ & $\begin{array}{l}\text { Se extrae de la hoja o penca y sirve para } \\
\text { curar: } \\
\text { - Gastritis, } \\
\text { - Diabetes, } \\
\text { - Granos en la cara } \\
\text { - Cicatrizar heridas } \\
\text { - Para aliviar la tos. Además, se emplea } \\
\text { en enfermedades del sistema digesti- } \\
\text { vo, endocrino, heridas y desórdenes } \\
\text { del sistema respiratorio, cutáneo, } \\
\text { inflamaciones (tomar el agua miel) } \\
\text { prostáticas. }\end{array}$ \\
\hline & & & $\begin{array}{l}\text { En base al gel que tienen las pencas } \\
\text { también se puede realizar cremas }\end{array}$ \\
\hline
\end{tabular}


Los magueyes se reproducen principalmente por los hijuelos que se desarrollan en la base del tallo de la planta madre, o bien por las semillas que produce la floración; ésta, que ocurre solamente una vez en la vida de un maguey y que es el anuncio de su muerte.

El estudio tiene como objetivo identificar los componentes químicos del jugo del maguey, tomado del hoyo que queda al centro del racimo de las pencas al extraerse el tallo, como un primer momento en la investigación y recabar información de creencias populares y explicaciones empíricas.

\section{MATERIAL Y MÉTODO}

Para el estudio de las propiedades químicas del maguey y las creencias populares de su acción curativa del jugo, llamado Upi (lengua quechua del lugar, que significa bebida), se desarrollaron entrevistas a los pobladores de la región, y análisis en laboratorio de las muestras líquidas llevadas refrigeradas.

En su primera etapa el proyecto tiene un nivel de estudio explorativo - descriptivo.

\section{RESULTADOS}

Se ha registrado informes de creencias populares que manifiestan casos de curaciones prostáticas, e inclusive entrevistado a personas mayores de la tercera edad que manifiestan haber superado la etapa final de cáncer con pronóstico de pocos meses de vida, casos que deben ser en un siguiente estudio analizados científicamente.

Se realizó el análisis en laboratorio de muestras del jugo del Maguey en cuestión.

\section{Tabla 2. Muestra de jugo de Maguey o Upi}

\begin{tabular}{lc}
\multicolumn{1}{c}{ Ensayo } & Resultados (\%) \\
\hline Proteínas $(\mathrm{g} / 100 \mathrm{~g}$ de muestra original) (factor 6.25$)$ & 0,7 \\
\hline Fibra (g/100g de muestra original) & 0,0 \\
\hline Grasa $(\mathrm{g} / 100 \mathrm{~g}$ de muestra original) & 0,0 \\
\hline Ceniza (g/100g de muestra original) & 0,6 \\
\hline Humedad (g/100g de muestra original) & 89,0 \\
\hline Carbohidratos (g/100g de muestra original) & 9,7 \\
\hline Sólidos solubles & 12,4 \\
\hline Energía total (g/100g de muestra original) & 41,6 \\
\hline Ph & 4,32 \\
\hline Fuente: Muestra enviada por la Dirección de Investigación de Universidad Alas Peruanas al laboratorio de la \\
Molina Calidad Total de la Universidad Nacional Agraria La Molina.
\end{tabular}

\section{DISCUSIÓN}

Se observa poca similitud entre los resultados de las muestras llevadas por nosotros al laboratorio y los resultados de los análisis hechos a la chancaca por otros investigadores (Dávila 2002)
(Producto obtenido del proceso de hervido del jugo del Maguey). Estas diferencias podrían ser resultado de pérdidas en los componentes químicos por el proceso de hervido del jugo luego sacado para la horma de chancaca. 


\section{Tabla 3. Análisis de la Chancaca}

\begin{tabular}{|c|c|}
\hline Humedad (g/100g de muestra original) & 11.76 \\
\hline Grasa (g/100g de muestra original) & 0.04 \\
\hline Carbohidratos (g/100g de muestra original) & 65.04 \\
\hline energía total (g/100g de muestra original) & 346.60 \\
\hline Proteínas (g/100g de muestra original) & 0.92 \\
\hline Fibra (g/100g de muestra original) & 0,17 \\
\hline Ceniza (g/100g de muestra original) & 1,64 \\
\hline Fósforo (mg/100g de muestra original) & 2.66 \\
\hline Hierro (mg/Kg de muestra) & 78.42 \\
\hline Calcio (mg/Kg de muestra) & 466.04 \\
\hline Magnesio (mg/Kg de muestra) & 796.15 \\
\hline Zinc (mg/Kg de muestra) & 4.17 \\
\hline Sodio (mg/Kg de muestra) & 1250 \\
\hline Potasio (mg/Kg de muestra) & 1481.82 \\
\hline Vitamina A (ug retinol/100g de muestra) & 50.00 \\
\hline Vitamina D (mg/100g de muestra) & 0.97 \\
\hline Vitamina C (mg/100g de muestra) & 18.60 \\
\hline Ácido Fólico (ug/100g de muestra) & 30.00 \\
\hline Vitamina B1 (mg/100g de muestra) & 0.15 \\
\hline Vitamina B2 (mg/100g de muestra) & 0.20 \\
\hline Vitamina B5 (mg/100g de muestra) & No detectable \\
\hline Vitamina B6 (mg/100g de muestra) & No detectable \\
\hline Vitamina B12 (mg/100g de muestra) & No detectable \\
\hline
\end{tabular}

Fuente: Tesis para optar el grado de Magíster Scientiae (Estrategias para la comercialización de los derivados de la Cabuya, Agave americana L.). César Dávila Veliz (2002). Univ. Nacional Agraria la Molina.

\section{BIBLIOGRAFÍA}

1. Dávila Véliz, César: Estrategias para la comercialización de los derivados de la Cabuya, (Agave americana L.). UNALM. Escuela de Post Grado. Tesis, 2002.

2. Deza R., Jaime, Pineda V., Elvis.: Una aproximación de Pisos Ecológicos y su Potencialidad Forrajero, Agrícola, Ganadero y otras Oportunidades
Económicas en Churcampa, Huancavelica. Rv. Ciencia y Desarrollo N ${ }^{\circ}$ : 83-101. Universidad Alas Peruanas. Dic. 2004 


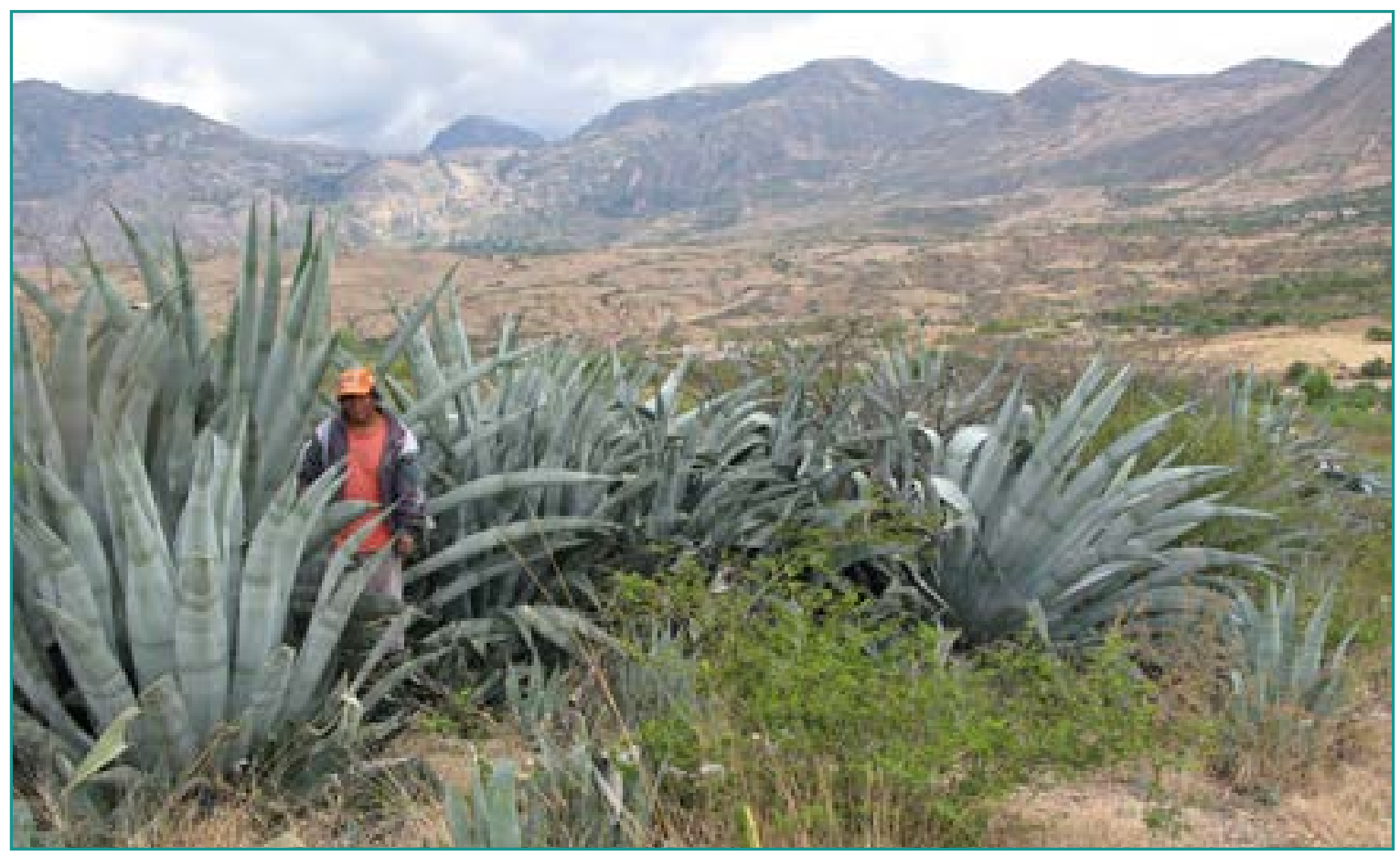

Figura 1: Campesino de Mayocc dedicado a la producción de "Upi”

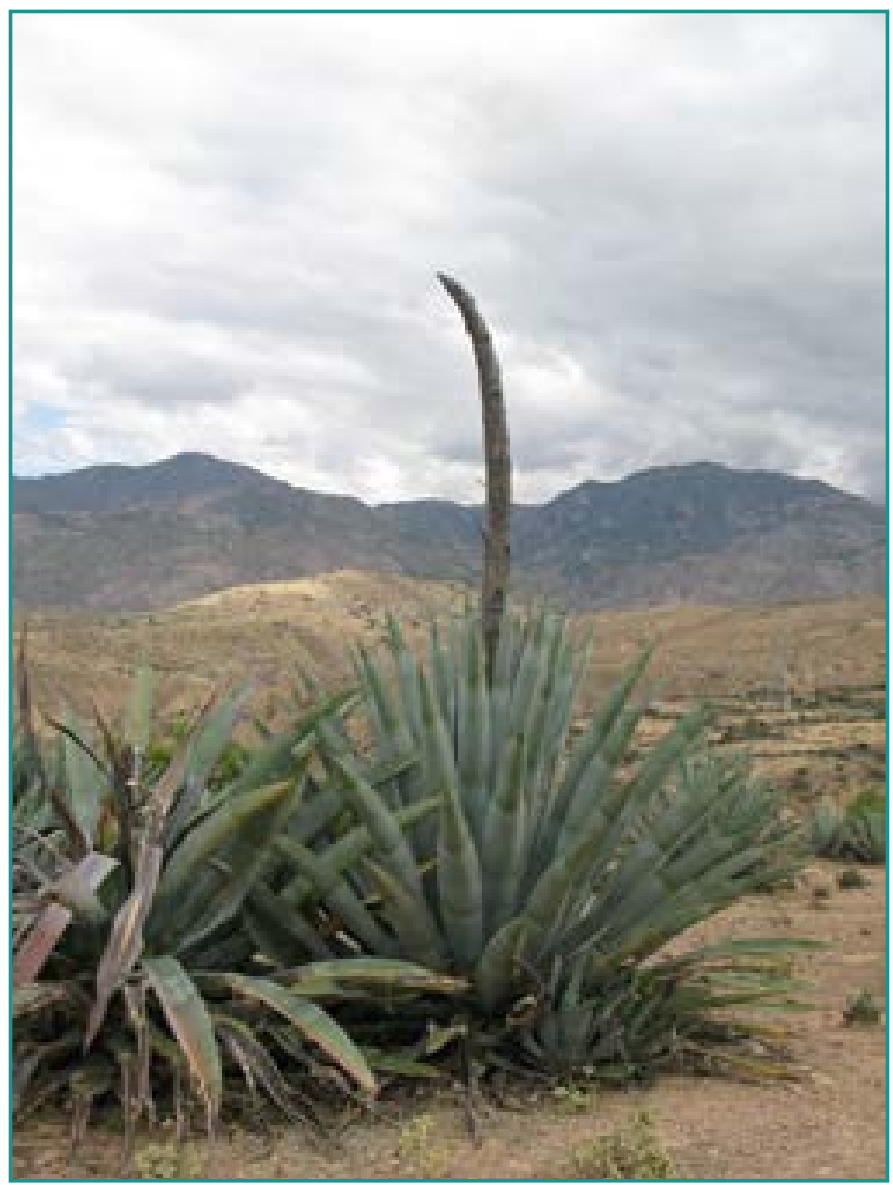

Figura 2: Planta silvestre de Maguey (Agave americana L.) 


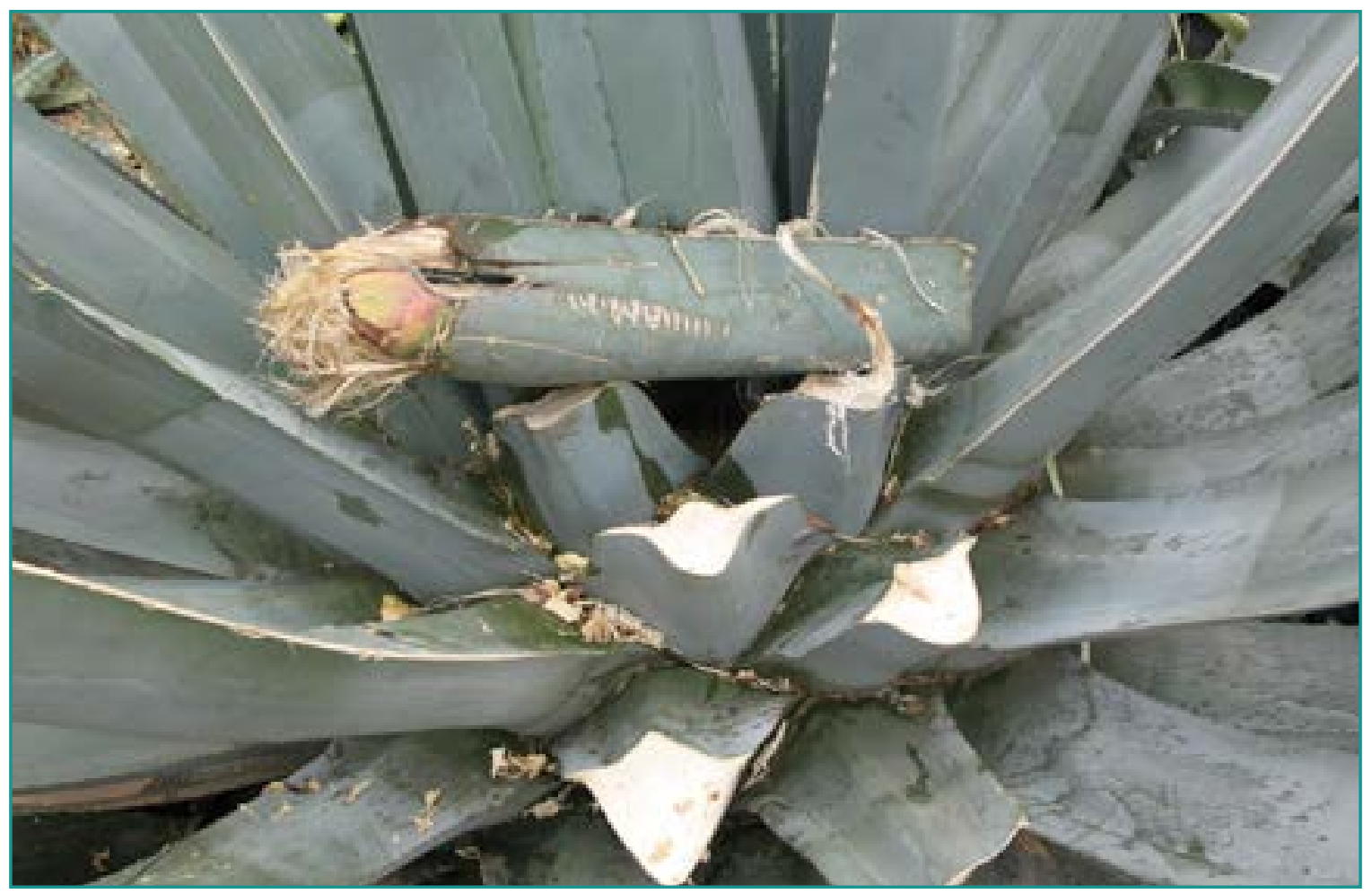

Figura 3: Depósito del “Upi” en el hoyo dejado al sacar el tallo.

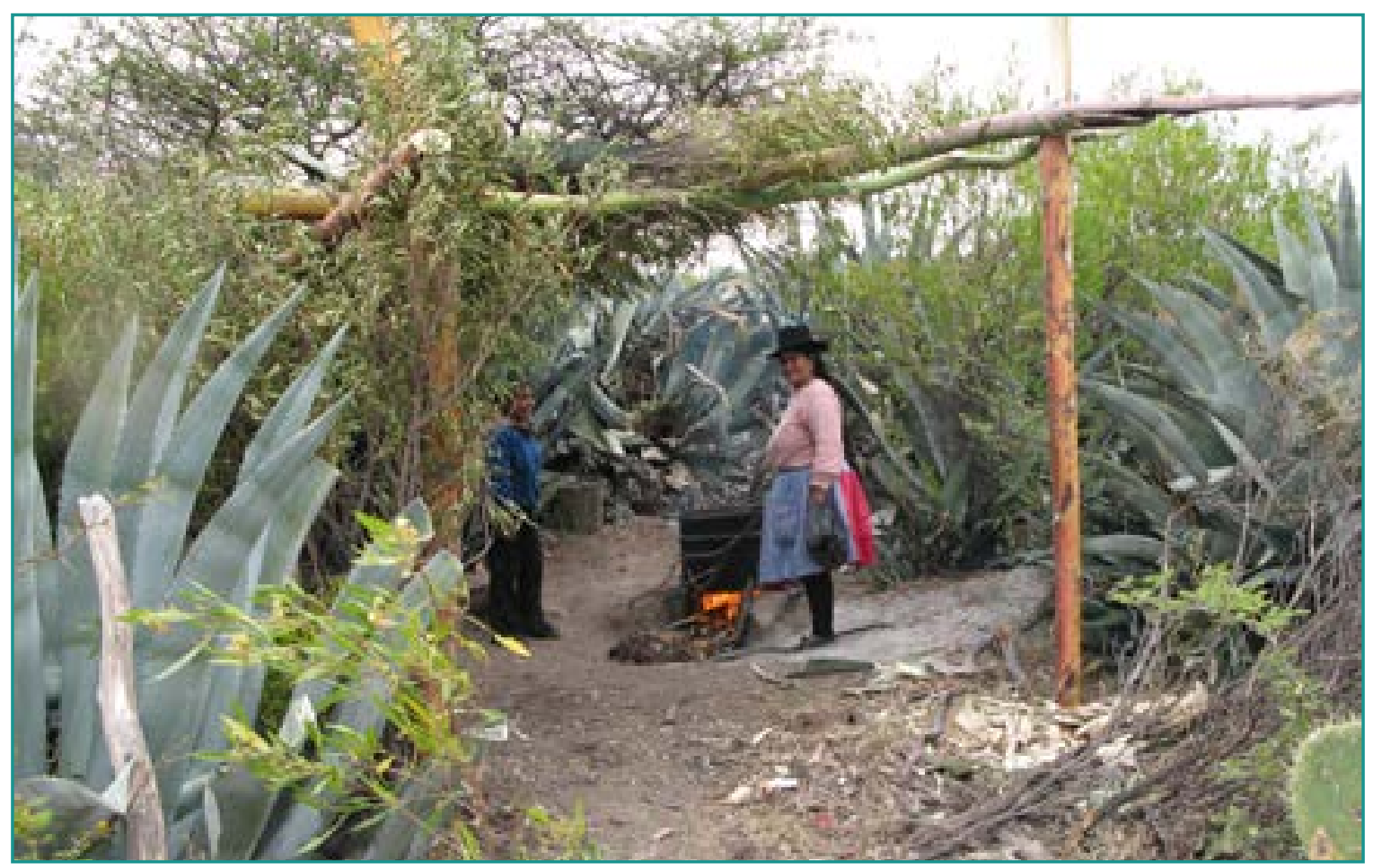

Figura 4: Hervido del "Upi" para hacer chancaca 


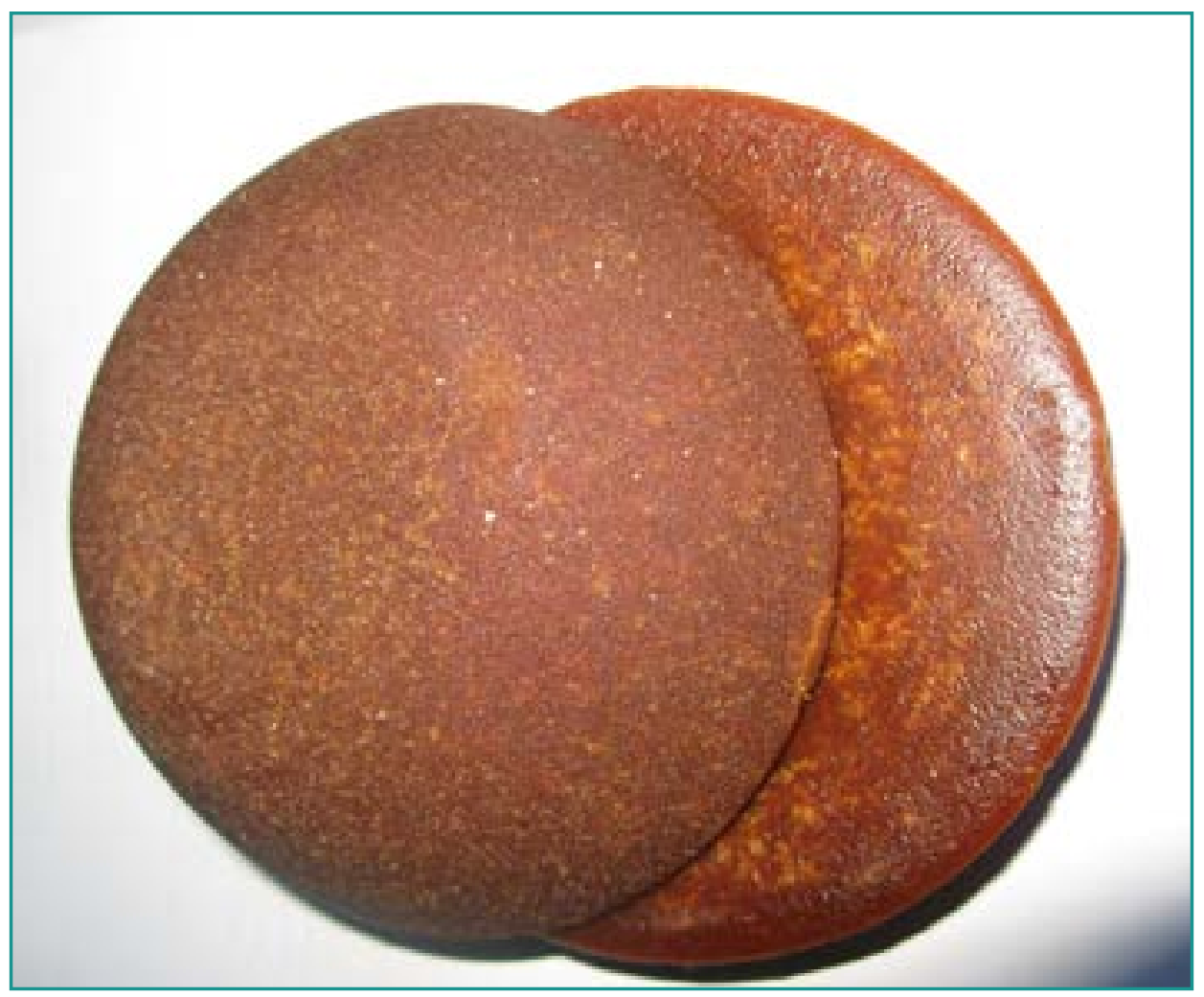

Figura 5: Piezas de chancaca de Maguey para la venta a los transeúntes

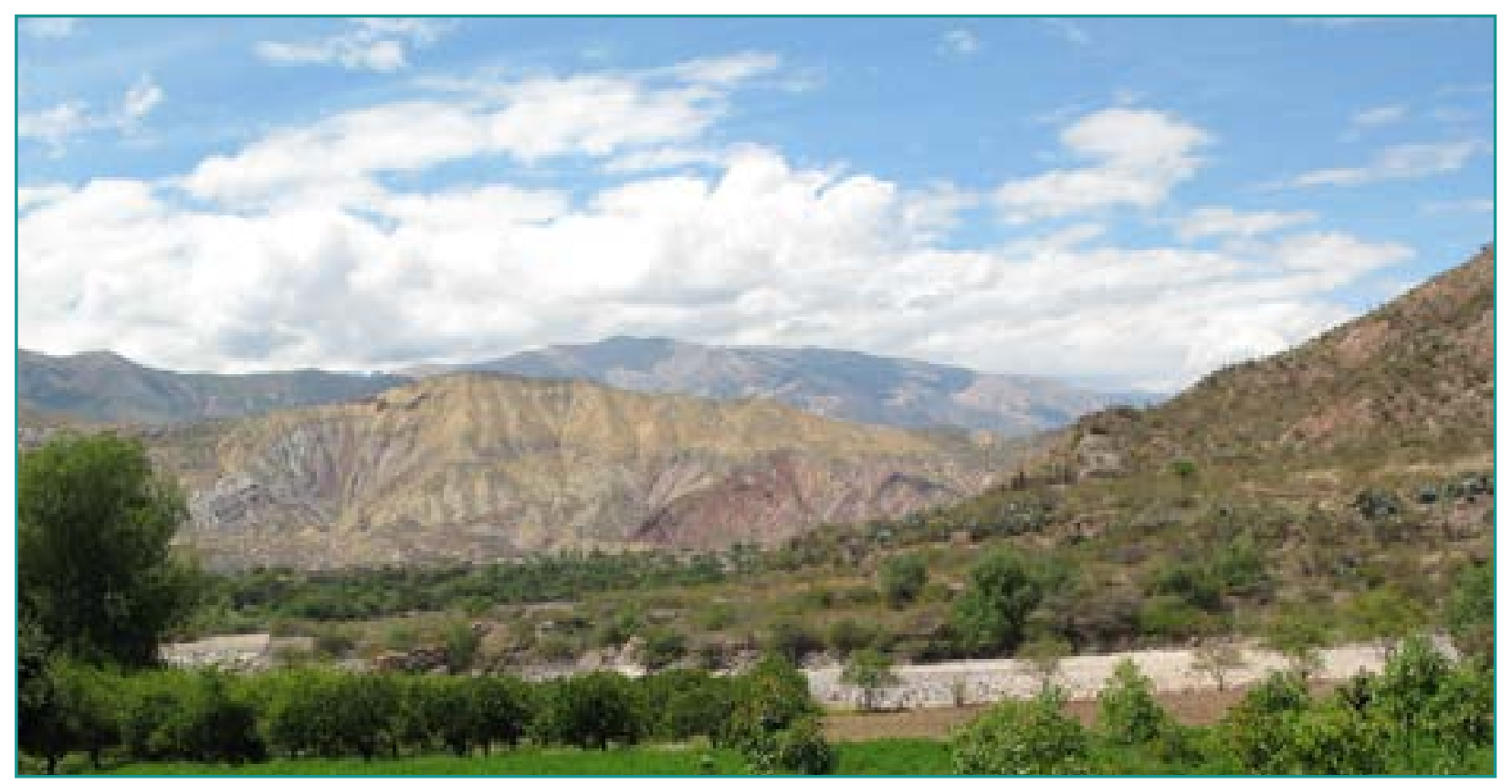

Figura 6: Vista general del lugar de producción 